\title{
8th International Congress on Biocatalysis (Biocat2016), Hamburg, Germany, 28 August-1 September, 2016
}

\author{
Skander Elleuche 1,2, Petra Esselun 1,2, Ralf Grote 1,2, Barbara Klippel 1,2, Anna Krüger 1,2, \\ Andreas Liese ${ }^{1,3}$, Margit Ostrop ${ }^{1,4}$, Christian Schäfers ${ }^{1,2}$, Carola Schröder ${ }^{1,2}$ and \\ Garabed Antranikian 2,5,* \\ 1 8th International Congress on Biocatalysis (Biocat2016)-Member of the local organizing committee, \\ Hamburg 21073, Germany; skander.elleuche@tuhh.de (S.E.); p.esselun@tuhh.de (P.E.); grote@tuhh.de (R.G.); \\ barbara.klippel@tuhh.de (B.K.); anna.krueger@tuhh.de (A.K.); liese@tuhh.de (A.L.); \\ ostrop@tutech.de (M.O.); christian.schaefers@tuhh.de (Ch.S.); carola.schroeder@tuhh.de (Ca.S.) \\ 2 Institute of Technical Microbiology, Hamburg University of Technology (TUHH), Hamburg 21073, Germany \\ 3 Institute of Technical Biocatalysis, Hamburg University of Technology (TUHH), Hamburg 21073, Germany \\ 4 TuTech Innovation GmbH, Hamburg 21079, Germany \\ 5 Biocat2016-Conference chair, Hamburg 21073, Germany \\ * Correspondence: antranikian@tuhh.de; Tel.: +49-(0)40-42878-3117; Fax: +49-(0)40-42878-2582
}

Academic Editor: Keith Hohn

Received: 11 October 2016; Accepted: 14 October 2016; Published: 18 October 2016

\begin{abstract}
The "8th International Congress on Biocatalysis (biocat2016)" is part of a biennial conference series. Biocatalysis is a topic based on the edge of biology and chemistry, which brings together scientists from the life sciences, engineers and computer scientists. This international conference serves as a platform to meet researchers from all over the world, to find collaboration partners for future projects and to gain novel insights into modern topics and techniques. Biocat covers the most exciting aspects and the latest developments in biocatalysis, including enzyme discovery, evolution and application, bioprocess engineering, cascade reaction systems and nanobiotechnology. In 2016, we welcomed 367 expert delegates in the respective fields. Established and young scientists from academia and the industry presented 51 lectures, 37 lightning talks and 234 posters. In addition, the biocat award, which is among the most prestigious awards in the field of biotechnology, has been awarded for the sixth time in the categories "Science in academia", "Lifetime achievement" and "Industry".
\end{abstract}

Keywords: bioprocesses; cascade reactions; enzyme discovery and engineering; enzymes; structure and function; sustainability

\section{Introduction}

Biocat was established in 2002 at the Hamburg University of Technology (TUHH) in Hamburg, Germany, under the direction of Garabed Antranikian, head of the Institute of Technical Microbiology and of the Hamburg University of Technology. Since then, eight congresses have been held in Hamburg, Germany, hosting more than 225 invited speakers and welcoming about 3000 participants from over 40 countries, including Australia, Austria, Brazil, China, the Czech Republic, Denmark, France, Germany, India, Ireland, Italy, Japan, Poland, Romania, Saudi Arabia, Singapore, Spain, Sweden, Switzerland, the Netherlands, Turkey, the United Kingdom, the United States of America and many more. The start of the conference series in 2002 until 2006 was supported through grants by the DBU ("Deutsche Bundesstiftung Umwelt"), and from 2008 to 2012 by the BMBF 
("Bundesministerium für Bildung und Forschung"), while the latter meetings of this well-established conference series are now completely self-financed.

Biocat2016 took place from 28 August to 1 September, 2016. The local organizing committee included members of the Institutes of Technical Microbiology and Technical Biocatalysis (both located at the TUHH). The congress was organized in cooperation with the Tutech Innovation $\mathrm{GmbH}$ and chaired by Antranikian. A total of 367 participants met at the conference venue, which is located on the campus of the TUHH (Figure 1).

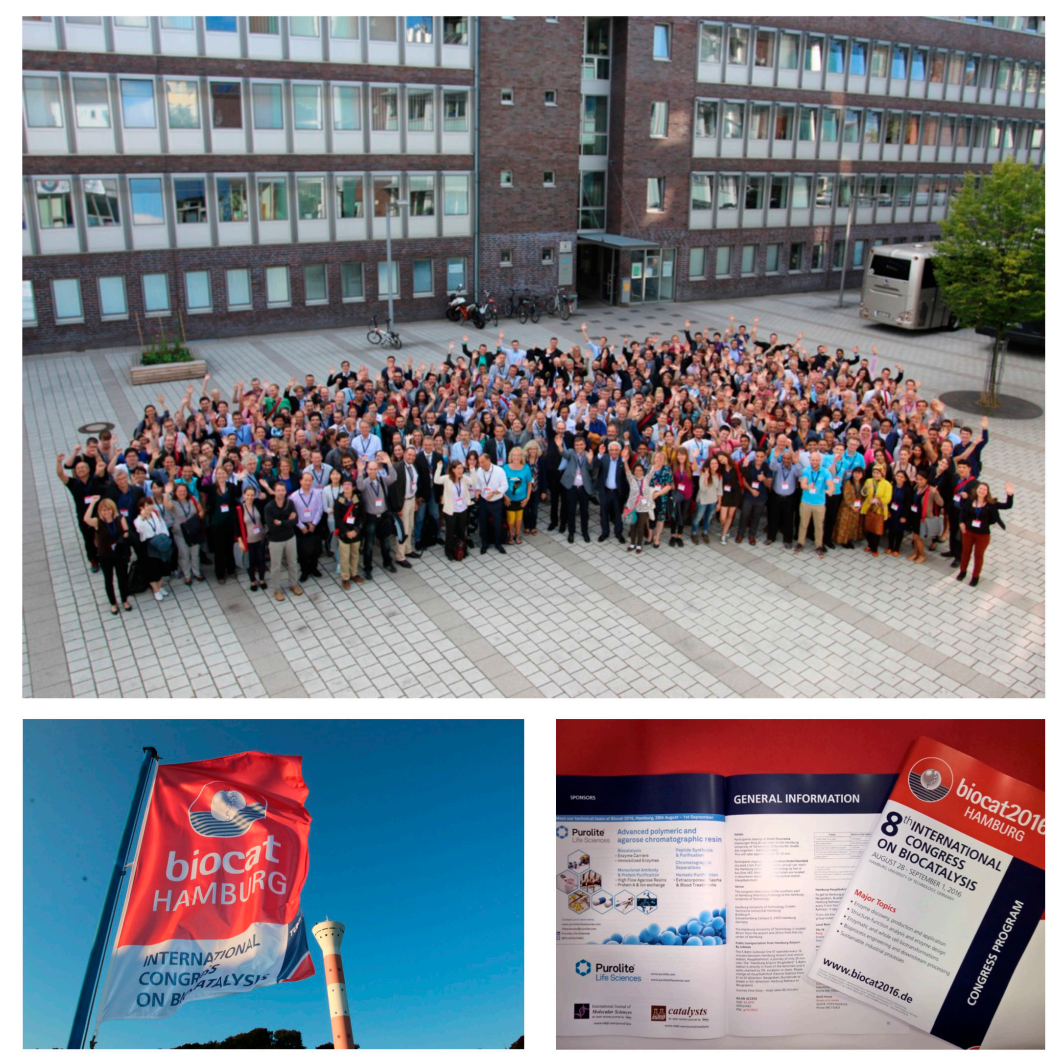

Figure 1. Impressions from the conference biocat2016. Top: Group picture. Bottom left: Biocat flag on board of a paddle wheel steamer on the Elbe River. Bottom right: Conference program (8th International Congress of Biocatalysis: Biocat2016, Book of Abstracts; Antranikian, G., Ed.; Tutech Innovation GmbH, Tutech Verlag: Hamburg, Germany, 2016; ISBN: 978-3-946094-07-4).

This conference series offers space for both well-established scientists to present their latest findings in the daily plenary lectures and oral sessions, and for young researchers to enter an international scientific atmosphere by giving oral presentations or the well-established short lightning talks. Moreover, biocat not only brings scientists together, but also serves as one of the most prominent and best platforms for diverse companies.

The successful concept of the biocat conferences is based on an interdisciplinary approach integrating the know-how of well-acknowledged biologists, biochemists, chemists, computer scientists and engineers from academia and industry. It serves as an international platform for the dissemination of the latest research results in a balanced program covering the following fields:

- Topic 1-Enzyme discovery, production and application

- Topic 2-Structure-function analysis and enzyme design

- Topic 3-Enzymatic and whole cell biotransformations

- Topic 4-Bioprocess engineering and downstream processing

- Topic 5-Sustainable industrial processes 
The focus of the sessions was versatile in order to shed light on a wide range of novel and important subjects and to offer outstanding opportunities for senior and young scientists. There were one opening and 17 plenary lectures, 33 competitively chosen talks, 37 short talks (lightning talks) and 234 posters. The format of lightning talks was established at biocat2014, giving some of the poster presenters the opportunity to briefly introduce their ongoing research topics to the audience. These short talks run end-to-end, with discussions taking place during the respective poster sessions. Biocat2016 had a number of student oral presentations and lightning talks and posters. Among them, 10 young scientists were awarded a poster prize to underline the quality of the subjects, experimental data and presentation.

\section{Highlights of the Conference}

On the first day of the congress, Garabed Antranikian, conference chair and president of the TUHH, hosted the opening ceremony (Figure 2). He introduced the audience to the history of the biocat conference series and the scientific program of the university. A music ensemble composed of Lisa Schmalz (soprano), Boglárka Pecze (clarinet) and Mariana Popova (piano) electrified the audience with a potpourri of classical and modern songs (Figure 2). Subsequently, Kurt Faber from the University of Graz held the distinguished opening lecture (Figure 2). Faber started with an overview of the "history of biocatalysis in a nutshell" and demonstrated the great potential of combining enzymes in a single reactor for utilization in reaction cascades. By showing applications for alcohol dehydrogenases, transaminases and monooxygenases, interesting alternative biocatalytic cascades for conventional chemical reactions were presented. The MDPI journals Catalyst and International Journal of Molecular Sciences supported Faber's participation at biocat2016 by offering a travel grant.
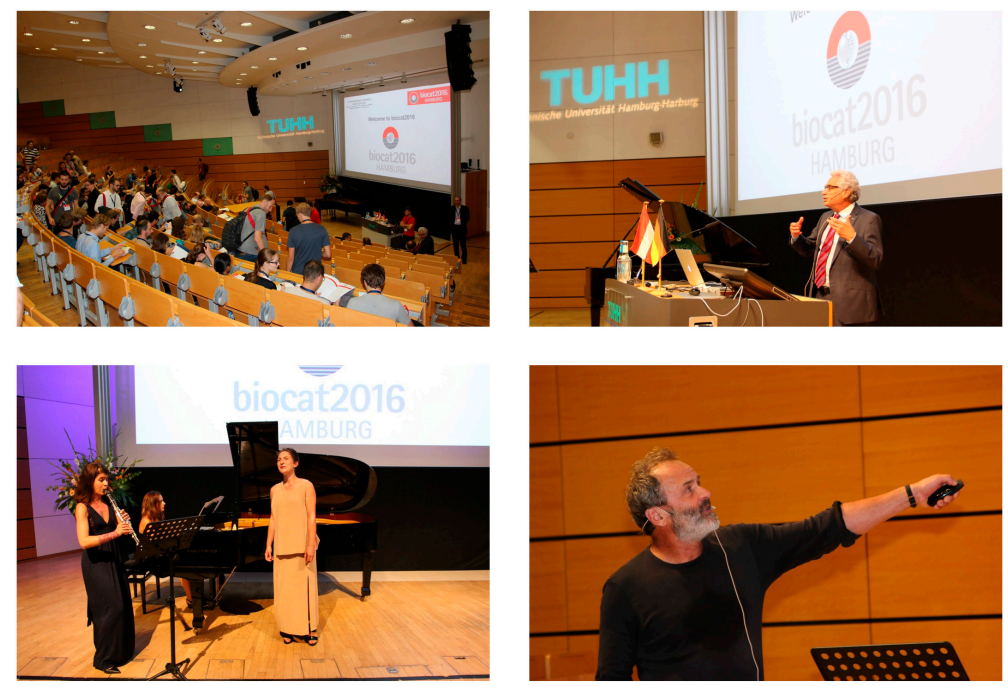

Figure 2. Biocat2016 opening ceremony. Top left: Audience. Top right: Congress chair Garabed Antranikian welcomes the participants. Bottom left: Musicians. Bottom right: Kurt Faber gives the prestigious opening lecture.

Internationally recognized scientists gave daily plenary sessions in the morning, followed by the main part of the conference program, which was devoted to open lectures (morning and afternoon sessions), lighting talks and poster sessions. There were no parallel lectures to offer each participant the opportunity to attend each high-level contribution. The program highlights of each topic and the special session on industrial implementations are briefly summarized below.

\subsection{Topic 1-Enzyme Discovery, Production and Application}

The demand for novel and improved biocatalysts is considered one major research field for the development of environmentally friendly and sustainable industry. This was the focus 
of the conference section on "Enzyme discovery, production and application". To achieve more attractive and efficient biocatalyzed processes, the knowledge of biocatalysts' structures and functions combined with innovative strategies may pave the way for a bio-based future. New sequence analysis platforms for the faster and more efficient discovery of novel enzymes as well as new chromogenic substrates for enlarged activity-based screening methods were proposed. Thereby, vast amounts of sequence data are generated on a daily basis. Lene Lange, from the Technical University of Denmark, presented a new and freely available technology platform to discover novel enzymes from large datasets. Function- and sequence-based technologies were also combined in further screening technologies. The authors agreed on the requirement of promoting optimized computational structure/function/specificity prediction for the efficient exploitation of nature's resources. In another keynote lecture, Colin Scott, CSIRO, Australia, presented a NanoFactory as a solution for a continuous multi-step system with a retained biocatalyst to recycle a required cofactor. Moreover, several solutions were proposed to face the environmental plastic issue, e.g., the development of enzymatically produced polyesters from bio-based monomers. Further interesting presentations paid attention to the optimization of the efficient production of secondary and tertiary amines, butadiene or chitooligosaccharides. Among others, lipolytic enzymes were emphasized for the generation of high-value esters. One intention of several experts in the field was the biodegradation or bioconversion of polysaccharides into high-value products for biotechnological applications. For that account, novel enzymes and their production were introduced to the audience. In this context, Lucia Gardossi, University of Trieste, emphasized the importance of multi-technology know-how to actuate the progress from conventional chemistry to biotechnology. All contributions were conducive to broadening our knowledge in the field of enzyme discovery, production and application for a future bio-based industry.

\subsection{Topic 2-Structure-Function Analysis and Enzyme Design}

The second major topic was headlined "Structure-function analysis and enzyme design", and all presented contributions covered a wide field of research subjects including proteomics, crystal structure analysis, directed evolution or synthetic biology. Altogether, 20 oral and lightning talks as well as 30 posters were presented during the conference covering this topic. Most of these contributions included either crystallization studies to solve crucial structural properties of industrially relevant biocatalysts or directed evolution approaches by protein design to maximize the performance, stability or selectivity of the enzyme of interest. Donald Hilvert, Swiss Federal Institute of Technology in Zürich, Switzerland, highlighted protein design approaches and explained what we can learn from nature's examples. He emphasized the importance of both computational and evolutionary approaches and suggested a combined strategy of these two approaches to gain engineered catalysts with better performances. Marco Fraaije, University of Groningen, the Netherlands, shared impressing insights of an applied approach during his talk about the discovery and engineering of robust oxidative biocatalysts. Furthermore, Isabelle André from the LISBP-INSA in Toulouse held another inspiring talk about "Computer-aided engineering of carbohydrate-active enzymes for novel synthetic reactions" and paid special attention to recent improvements in the development of programmed chemo-enzymatic pathways. The selectivity of enzymes, especially the enantioselectivity of given reactions that are most relevant for industrial applications, was another well-discussed subtopic at biocat2016. Among others, the well-known scientists Loredano Pollegioni, Kai Tittmann and Manfred Reetz shared novel insights during their talks. Altogether, this year's trend is clearly to take the best enzymes on the market and make them even better. Nowadays, many datasets are available, including genomics, metagenomics, proteomics, secretomics or structural-based datasets. The take-home message emphasized using these datasets and transferring the knowledge into protein design approaches to engineer improved biocatalysts. 


\subsection{Topic 3-Enzymatic and Whole Cell Biotransformations}

The combination of oxidation and reduction reactions in a cascade allows performing transformations in an economic and efficient way. The utilization of different enzymes and metabolically engineered microorganisms for this purpose was demonstrated in this section. Another emphasis was placed on direct in situ, coupled reaction steps in one cascade for the biocatalytical synthesis of chemical intermediates, building blocks and fine chemicals. In contrast to conventional chemical routes, high enantioselectivity is achieved in enzyme-mediated reactions. The design of novel cascades by combining various types of enzymes is another advantage of using chemo-enzymatic cascade reactions. The lecturers of topic 3 paid special attention to enzymatic activities of alcohol dehydrogenases, transaminases, lyases and monooxygenases. Beside these biocatalysts, the utilization of transferases is of major interest. Bernd Nidetzky, Graz University of Technology, Austria, demonstrated the potential of glycosyltransferases used for the synthesis of glycosides. However, not only glycosyltransferases are able to catalyze transferase reactions-hydrolases (lipases, glycoside hydrolases) are also promising candidates for the catalysis of such reactions when hydrolytic activity is suppressed. The take-home message of this topic was that reaction cascades and the combination of bio- and chemo-catalysis in one pot offer new routes for the synthesis of novel substances and their derivatives and have great potential to achieve high enantio- and stereo-selectivities.

\subsection{Topic 4-Bioprocess Engineering and Downstream Processing}

A prerequisite for the successful application of biocatalysts in industry is the development of novel biotechnological processes. Therefore, an important topic of the conference was "Bioprocess engineering and downstream processing". Major trends within this topic, also discussed by several speakers, were cascade reactions, novel enzyme immobilization techniques and compartmentalization. Sven Panke, Swiss Federal Institute of Technology in Zürich, Switzerland, presented an elegant novel bioprocess for the production of a rare sugar by applying a three-step enzyme cascade reaction coupled with simulated moving bed technology. Cristina Carucci, University of Limerick, Ireland, highlighted the importance of novel enzyme immobilization methods to enable recycling of expensive biocatalysts. In her talk, she proposed the application of metal-organic materials for in situ immobilization of a wide range of enzymes. A different immobilization technique for application in enzymatic microfluidic devices was presented by Christian Hoffmann, Technical University of Denmark, Denmark. He gave insights into the development of off-stoichiometric thiol-ene networks for covalent immobilization of enzymes in microreactors. Finally, Kathrin Castiglione, Technical University of Munich, Germany, proposed a sophisticated strategy for the production of nano-scale enzyme membrane reactors also comprising immobilized enzymes.

\subsection{Topic 5-Sustainable Industrial Processes}

The need to maximize resource efficiency, conserve energy and non-renewable resources, prevent risks, pollution and minimize waste in an efficient and environmentally friendly production was emphasized in another main topic of the conference: "Sustainable industrial processes". Speakers from various biotechnological companies highlighted the importance of applying sustainable processes for the synthesis of important biotechnological products, such as active pharmaceutical ingredients or building blocks. Riccardo Motterle, F.I.S. (Fabbrica Italiana Sintetici), Italy, presented the benefits of a design of an experimental approach for optimization of biocatalytic synthesis of testosterone and a statin building block. In his talk, "Putting the bottle on the table", Philipp Süss, Enzymicals AG, Germany, reviewed the process development and optimization of chemo-biocatalytic reactions starting from enzyme screening to finally "putting the product on the table". Finally, Jessica Rehdorf, BRAIN AG, Germany, challenged the audience with her presentation about sustainability evaluation of (bio)processes. She pointed out that sustainability is a key parameter for the bioeconomy movement, but that bioprocesses are not per se environmentally friendly or "green". Furthermore, she gave 
insights into a case study of an antimicrobial compound showing different tools and metrics to perform the environmental assessment of a process.

\subsection{Special Session-Industrial Implementations}

The congress also included a plenary session focused on "Industrial implementations". This special session comprised lectures from representatives of global players in the biotechnological industry. The four invited speakers discussed recent developments concerning enzyme and process optimization and the application of enzyme cascades. In his talk, Jens Erik Nielsen, Novozymes A/S, Denmark, addressed two interwoven problems: structure-based protein engineering and large datasets. The generation and exploitation of experimental data in order to produce tailor-made enzymes for biotechnological applications is one of the major challenges in biocatalysis. Martin Schürmann, DSM, the Netherlands, focused on the sustainability of processes to synthesize chemical building blocks and intermediates from renewable resources, which can only be achieved by multidisciplinary approaches including biocatalysis as well as chemical syntheses. In order to further strengthen biocatalytic approaches is the generation of platforms containing enzymes with broad substrate scope and high activity. The latter was also seized by Oliver Thum, Evonik Creavis GmbH, Germany, who compared the biocatalytic functionalization of cheap and simple starting materials for the production of biopolymers with the search for the philosopher's stone. In his lecture on "Efficient synthesis of pharmaceutical compounds using biocatalysis", John Wong, Pfizer, USA, also emphasized the importance to generate sustainable processes by applying novel enzymes as a powerful tool towards this goal.

Another highlight of this year's congress was the biocat award ceremony, which traditionally takes place on a paddle wheel steamer on the Elbe River. This prestigious prize is awarded in three different categories (Figure 3).

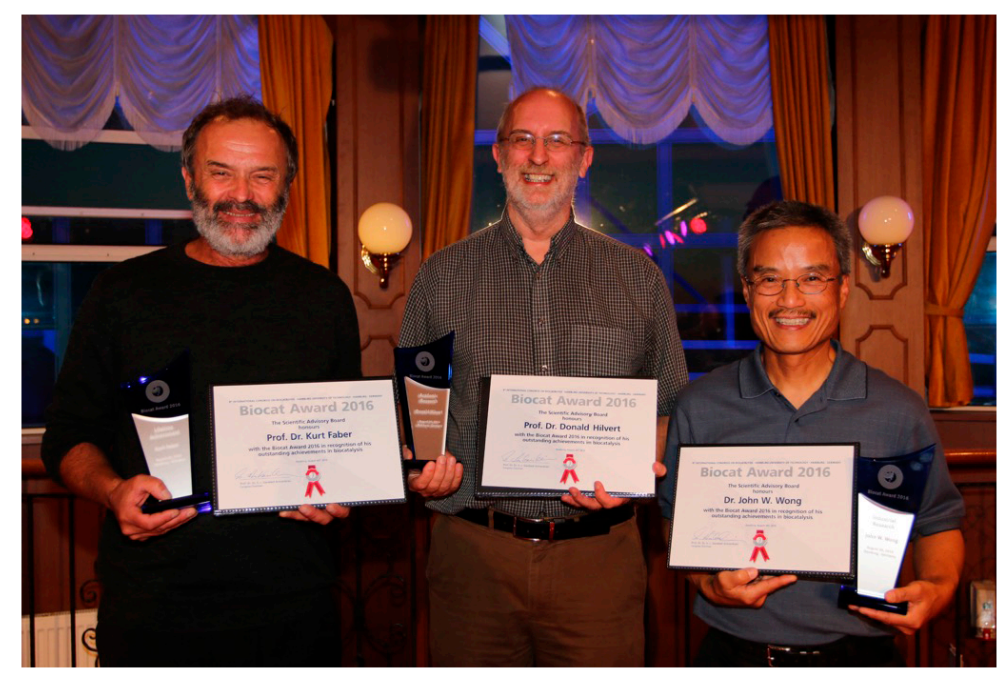

Figure 3. Biocat2016 awardees. Left: Kurt Faber received the biocat award for "Lifetime achievement". Middle: Donald Hilvert was awarded in the category "Science in academia". Right: John Wong was honored with the biocat award in the category "Industry".

Kurt Faber received the biocat award for "Lifetime achievement" for his outstanding career. He is a full professor at the University of Graz and a major contributor to the biocat series, including being a long-time member of the scientific advisory board. He gave about 600 lectures and poster contributions at scientific conferences and is the author of more than 300 publications. Faber made major contributions in the selective biotransformation of non-natural compounds and asymmetric synthesis of bioactive organic compounds. 
The biocat award in the category "Science in academia" went to Donald Hilvert. Since 1997, he has been a professor in the Laboratory of Organic Chemistry at the Swiss Federal Institute of Technology in Zürich, Switzerland. Hilvert received multiple scientific honors and awards, including Doctor of Philosophy honoris causis, Uppsala University, Sweden; he gave the prestigious Feodor Lynen Lecture and is a fellow of the American Academy of Arts and Sciences. Moreover, he is the author of about 240 scientific publications (e.g., in Nature, Science, PNAS and JACS) and a well-known expert in enzymology, enzyme engineering, molecular evolution and chemical biology.

The renowned scientist and industry representative John Wong, Pfizer, USA, was awarded the biocat prize in the category "Industry". Wong received his PhD from Simon Fraser University in Canada before progressing on the career ladder at Pfizer, USA. He was a Senior Research Scientist, Senior Research Investigator, Principal Research Investigator and Associate Research Fellow. Currently, Wong is Research Fellow at the Biocatalysis Center of Emphasis, Chemical Research and Development, Pfizer. He has broad experience in the pharmaceutical industry, working on biocatalysis, and a strong background in organic synthesis. During his long career, he developed chemo-enzymatic syntheses of target molecules, identified insect pheromones and elucidated versatile biosynthetic pathways to produce valuable chemical and pharmaceutical compounds.

\section{Conclusions}

In the last two decades, the biocat conference series became a forum for debate and discussion of the latest research activities in the field of biocatalysis. The scientific areas covered by the conference were those generally encountered by biocatalysts, including bioprocess engineering, biotransformation, protein structure determinations and enzyme discovery. A trend towards cascade reactions and nanobiotechnology has been noticed, but also the classical topics including enzyme engineering and the development of sustainable industry remain hot topics that are covered by various research groups from all over the planet. Although some riddles were solved, many unexplained problems in the field of biocatalysis still remain which demand further congresses. Moreover, the outstanding quality of all scientific presentations and the fruitful discussions underlined the degree of relevance of this biennial conference series. Therefore, we welcome you to attend the next biocat, which will take place in Hamburg from August 26 to 30, 2018. Please check http:/ / www.biocatconference.de for updates and further information.

Acknowledgments: All members of the Institutes of Technical Microbiology and Technical Biocatalysis as well as members of the TuTech Innovation $\mathrm{GmbH}$ and the members of the scientific advisory board are thanked for their valuable contribution.

Author Contributions: All authors were members of the local organizing committee and directly participated in the organization of the conference. G.A. is the congress chair of the biocat conference series. S.E. drafted the manuscript with the help of B.K., A.K., Ch.S. and Ca.S. All authors read and approved the final manuscript.

Conflicts of Interest: We declare potential conflicts of interest, because the authors are organizers and participants of the conference "biocat2016".

(c) 2016 by the authors; licensee MDPI, Basel, Switzerland. This article is an open access article distributed under the terms and conditions of the Creative Commons Attribution (CC-BY) license (http://creativecommons.org/licenses/by/4.0/). 